\title{
Modelling of Water Levels in a Reservoir using Modelica
}

\author{
Willem Mejer Dietmar Winkler \\ Department of Electrical Engineering, Information Technology and Cybernetics \\ University of South-Eastern Norway, Porsgrunn, Norway \\ willem.meijerdskagerakenergi.no, dietmar.winklereusn.no
}

\begin{abstract}
The regulation of hydro power plants is influenced by amongst others reservoir capacity and the knowledge about how it is influenced by changes in in- and outflow. The hydro power reservoir Aurdalsfjord has a small capacity compared to the inflow, a narrowing between inflow in the north and outflow in the south and has implemented strict governmental restriction.

The paper will present a reservoir model that was developed in order to investigate the water flow and water level conditions in Aurdalsfjord. The water level in each cell is described with a mass balance, while the flow between two cells is described with a momentum balance. The balance equations are formulated as DAEs. The model is developed in Modelica which allows it to be used in connection with models of a wide variety of other components that are present in a hydro power system. The model will be integrated in the open-source hydro power library OpenHPL developed at USN.

The Modelica model shows promising results. For Aurdalsfjord it needed to be adjusted with non-physical parameters. The reservoir model should be implemented for a less complex shaped reservoir in order to see if the challenges are related to the Modelica implementation or Aurdalsfjords shape.
\end{abstract}

Keywords: modelling, hydro power, reservoir dynamics, Modelica, OpenHPL

\section{Introduction}

This paper is based on a Master's Thesis (Meijer 2020) from the spring of 2020 at the University of South-Eastern Norway (USN). A dynamic reservoir model was developed with Modelica in order to investigate time-constants between in- and outflow in the hydro power reservoir Aurdalsfjord. The paper will present the Modelica model and use the Aurdalsfjord as an example in order to present some simulation results.

\subsection{Hydro Power}

Hydro power represents the main energy source in the Norwegian electricity supply and is the most important sustainable energy source worldwide. It transforms the potential and kinetic energy of flowing or falling water into electrical energy.
The regulation of hydro power plants is influenced by electricity prices, inflow, reservoir capacity and governmental restrictions. The relation between reservoir capacity and inflow decides the flexibility of the power plant. If the reservoir is small compared to the inflow, the inflow is more important and the power plant less flexible. If the reservoir however is large compared to the inflow, the amount of stored water is more important.

For some reservoirs these factors prove more challenging than others due to their capacity, shape or strict governmental restrictions. Aurdalsfjord is one example. It is the intake reservoir to the power plant Bagn, which is operated by the Norwegian hydro power company Skagerak Kraft AS. Its capacity is small compared to the inflow (received from two other power plants), has a narrowing between inflow in the north and outflow in the south and has implemented strict governmental restriction.

\subsection{Modelica}

Modelica is an object-oriented modelling language. It is designed to implement dynamic models equation systems as differential and algebraic equations (DAE). In contrast to most programming languages, Modelica translates the code to its most optimal state in C-code before simulation. Hence, the order in which the equations are written is not relevant. Models from a high variety of engineering fields can be connected, which makes it possible to develop full hydro power plants models.

Modelica models can be used with an optimisation and automation infrastructure using the python application interface OMPython (Open-Source Modelica Consortium 2020a). It is part of the open-source OpenModelica installation (Open-Source Modelica Consortium 2020b).

\subsection{Goal and Scope}

The dynamic reservoir model is developed that takes the in- and outflows as inputs and gives the water levels as output. The models purpose is to investigate water flows and levels in hydro power reservoirs under different in- and outflow situations. The model dynamics will be described by mass and momentum balances as DAEs.

The model will be integrated into the open-source hydro power library, OpenHPL (TMCC, USN 2020). OpenHPL is developed by USN for teaching and research purposes. The model is developed with OpenModelica, simulated with OMPython and tested with Dymola (Dassault Sys- 
tèmes 2020).

\section{Mathematical Description}

The flow in a reservoir can be described with a combination of mass and momentum balances. The reservoir needs to be discretised into a grid, where the water level in each cell is described with a mass balance and the flow between two cells is described with a momentum balance. Only horizontal flows are taken into account, vertical flows are neglected, as it is assumed that horizontal flow is significantly larger than vertical flow.

The main purpose of the model is to describe the water level in each of the cells. The input parameters are the in- and outflows at the borders. A block diagram for the general model is shown in Figure 1.

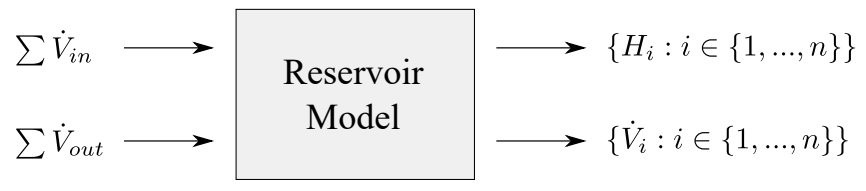

Figure 1. Block diagram for the model.

A DAE is a set of equations containing both ODEs and algebraic equations containing both variables and parameters. The ODEs describe the change of a quantity in time, while the algebraic equations describe the quantities in the ODEs by other variables and/or parameters. In order to be solvable, the DAE needs the same number of equations as variables.

In order to describe the equations, mass balance control volumes and momentum balance control volumes are introduces. The control volumes contain variable (mass or momentum) which changes within a limited volume. The change is caused by forces within the control volume or flow across the borders. The mass balance control volumes are the same as the reservoir cells, while the momentum balance control volumes cover half of one cell and half of the next.

\subsection{Mass Balance}

The mass balance describes the change of mass in a control volume over time. The change is equal to the mass flow into the control volume subtracted by the mass flow out of the control volume, as shown in the Equation 1. It is assumed that there is no internal mass conversion, as this is only relevant for nuclear reactions.

$$
\frac{\mathrm{d} m}{\mathrm{~d} t}=\dot{m}_{\mathrm{in}}-\dot{m}_{\mathrm{out}}
$$

The goal of the mass balance equation is to find the water level in the control volume as a function of the volume flow. Hence, the mass and mass flows need to be described by the algebraic equations in Equation 2 .

$$
\begin{aligned}
m & =\rho \cdot \Delta x \cdot \Delta y \cdot h \\
\dot{m}_{\text {in }} & =\rho \dot{V}_{\text {in }} \\
\dot{m}_{\text {out }} & =\rho \dot{V}_{\text {out }}
\end{aligned}
$$

In the reservoir model, each control volume has borders in both $\mathrm{x}$ - and y-direction, as shown in Figure 2. Hence, the mathematical description needs to be modified to fit two flow directions. The in and out indices are replaced by using positive and negative flow directions. The complete mass balance is described with Equation 3.

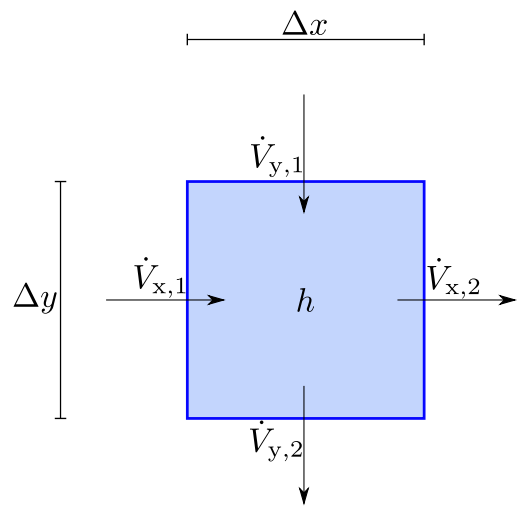

Figure 2. Mass balance control volume seen from above. The arrows show positive flow direction.

$$
\begin{aligned}
\frac{\mathrm{d} m}{\mathrm{~d} t} & =\dot{m}_{\mathrm{x} 1}+\dot{m}_{\mathrm{y} 1}-\dot{m}_{\mathrm{x} 2}-\dot{m}_{\mathrm{y} 2} \\
m & =\rho \cdot \Delta x \cdot \Delta y \cdot h \\
\dot{m}_{\mathrm{x} 1} & =\rho \cdot \dot{V}_{\mathrm{x} 1} \\
\dot{m}_{\mathrm{x} 2} & =\rho \cdot \dot{V}_{\mathrm{x} 2} \\
\dot{m}_{\mathrm{y} 1} & =\rho \cdot \dot{V}_{\mathrm{y} 1} \\
\dot{m}_{\mathrm{y} 2} & =\rho \cdot \dot{V}_{\mathrm{y} 2}
\end{aligned}
$$

\subsection{Momentum Balance}

The momentum balance describes the change in momentum over time in a control volume as a function of momentum flow in and out of the system in addition to the sum of all forces acting on the flow, see Equation 4.

$$
\frac{\mathrm{d} M}{\mathrm{~d} t}=\dot{M}_{\text {in }}-\dot{M}_{\text {out }}+\sum F
$$

In order to connect momentum to volume flow, the algebraic equations in Equation 5 are introduced.

$$
\begin{aligned}
M & =m \cdot v \\
m & =\rho \cdot A \cdot \Delta l \\
v & =\frac{\dot{V}}{A}
\end{aligned}
$$


The momentum flow can be described as a function of volume flow in and out of the system as shown in Equation 6 .

$$
\begin{aligned}
\dot{M}_{\text {in } / \text { out }} & =\dot{m}_{\text {in } / \text { out }} \cdot v_{\text {in } / \text { out }} \\
\dot{M}_{\text {in } / \text { out }} & =\rho \cdot \dot{V}_{\text {in } / \text { out }} \\
v_{\text {in } / \text { out }} & =\frac{\dot{V}_{\text {in } / \text { out }}}{A_{\text {in } / \text { out }}}
\end{aligned}
$$

The sum of the forces is given by the forces parallel to the horizontal flow. The only two forces which are taken into account are the pressure and the friction forces. It can be shown that they can be given by Equation 7 (pressure force) (Plumb 2014) and Equation 8 (friction force) (Lie 2017).

$$
\begin{gathered}
F_{\mathrm{p}}=\rho g \Delta w \frac{h_{1}^{2}-h_{2}^{2}}{2} \\
F_{\mathrm{f}}=\Delta l \rho g A S_{\mathrm{fs}} \\
S_{\mathrm{fs}}=k_{\mathrm{s}}^{2} \dot{V}^{2} \frac{\phi}{A^{3}}
\end{gathered}
$$

The friction slope, $S_{\mathrm{fs}}$ is introduced as part of the friction force. The wetting perimeter, $\phi$, is the total circumference of the cross section that produces friction. Due to the model containing mainly cells in the centre of the grid (not without borders), it is assumed that the wetting perimeter is equal to only the width, $\Delta w$.

In contrast to the mass balance, the momentum balance is only applicable in one flow direction. Hence, $\Delta w$ and $\Delta l$ equals $\Delta x$ and $\Delta y$ depending on the direction. Figure 3 shows a control volume for a momentum balance. The complete momentum balance is described with Equation 9. In order to take direction into account, all squared flows are split into a product of the flow times the absolute value of the flow.

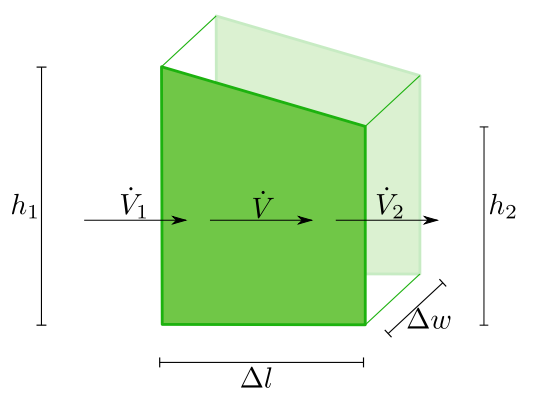

Figure 3. Momentum balance control value.

$$
\begin{aligned}
\frac{\mathrm{d} M}{\mathrm{~d} t} & =\dot{M}_{1}-\dot{M}_{2}+F_{\mathrm{p}}-F_{\mathrm{f}} \\
m & =\rho \cdot \Delta l \cdot \dot{V} \\
\dot{M}_{1} & =\rho \frac{\dot{V}_{1}\left|\dot{V}_{1}\right|}{A_{1}} \\
\dot{M}_{2} & =\rho \frac{\dot{V}_{2}\left|\dot{V}_{2}\right|}{A_{2}} \\
F_{\mathrm{p}} & =\rho g \Delta w \frac{h_{1}^{2}-h_{2}^{2}}{2} \\
F_{\mathrm{f}} & =\Delta l \rho g \bar{A} S_{\mathrm{fs}} \\
S_{\mathrm{fs}} & =k_{\mathrm{s}}^{2} \dot{V}|\dot{V}| \frac{\phi}{\bar{A}^{3}}
\end{aligned}
$$

The two sides of the momentum balance control volume can contain different depth values and be redrawn as Figure 4. Due to only horizontal flow being taken into account, the bottom part does not influence the system. The heights $h_{1}$ and $h_{2}$ in the pressure force (Equation 7) need to have the same reference point. Hence, Figure 3 shows only the upper part of the control volume.

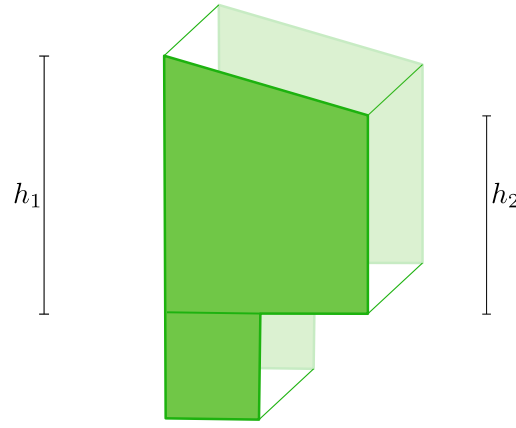

Figure 4. Different depth values.

There can occur situations where the level at one side is lower than the floor on the other side, as shown in Figure 5. In this case there is only flow from the top side. The adjustments in Equation 10 are made in order for Equation 9 to make sense. The reverse can also occur (left side below right side floor).

$$
\begin{aligned}
\dot{M}_{2} & =0 \\
h_{2} & =0
\end{aligned}
$$

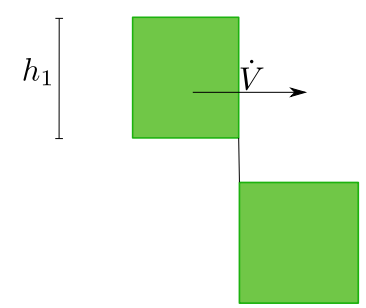

Figure 5. Case where water level on one side is below floor on other side. 


\subsection{Connecting Mass and Momentum Balance}

In order to describe the complete dynamics in the system three stacked grids are needed, where the grids contain control volumes for:

- Mass balance

- Momentum balance in $\mathrm{x}$-direction

- Momentum balance in y-direction

The three grids are connected through the mass balance control volumes, which receive mass flows described by both momentum balance grids ( $\mathrm{x}$ - and $\mathrm{y}$-directions). The momentum balance control volumes receive water levels from the mass balance grid. Figure 6 shows a 1dimensional version of a stacked grid.

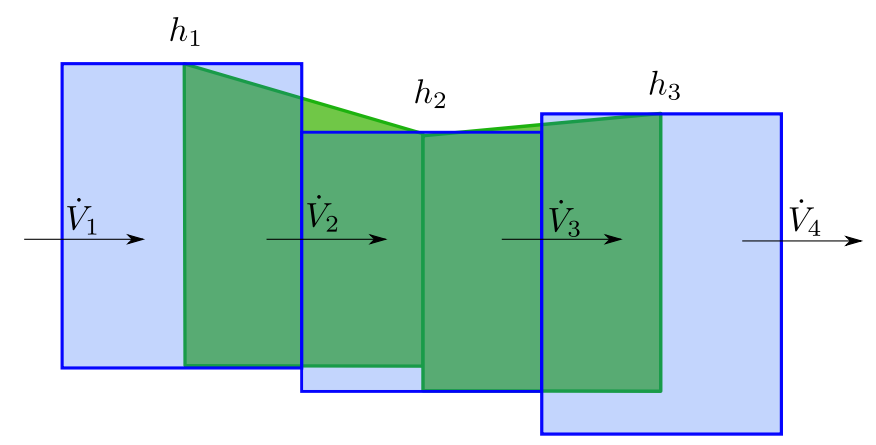

Figure 6. Mass balance (blue) and momentum balance (green) grids stacked on top of each other.

\section{Implementing in Modelica}

The goal of the reservoir model is to implement it together with in- and outflow components. The implementation of Aurdalsfjord is shown in Figure 7. The in- and outflow models read data from a csv-file. The data record (yellow box) contains values for relevant constants (e.g., $\left.g, p_{\mathrm{a}}\right)$ and is a functionality from OpenHPL.

This section will provide a description of how the main model, Reservoir, is implemented. The description is focused on the use of the model and the basic ideas behind the implementation. In addition, it is described how the model can be used to build more complex models, and simulation results are shown.

\subsection{Reservoir}

The reservoir model describes the water levels in the reservoir by receiving the in- and outflow as the inputs. Due to the model being implemented in Modelica as DAEs, all other values calculated along the way, e.g., volume flows between cells, are also available outputs.

\subsubsection{Parameters}

The parameters with explanations are shown in Figure 8. Some of the parameters are further explained.

depth is an array containing the depth values for the reservoir cells. The amount of cells is also (indirectly)

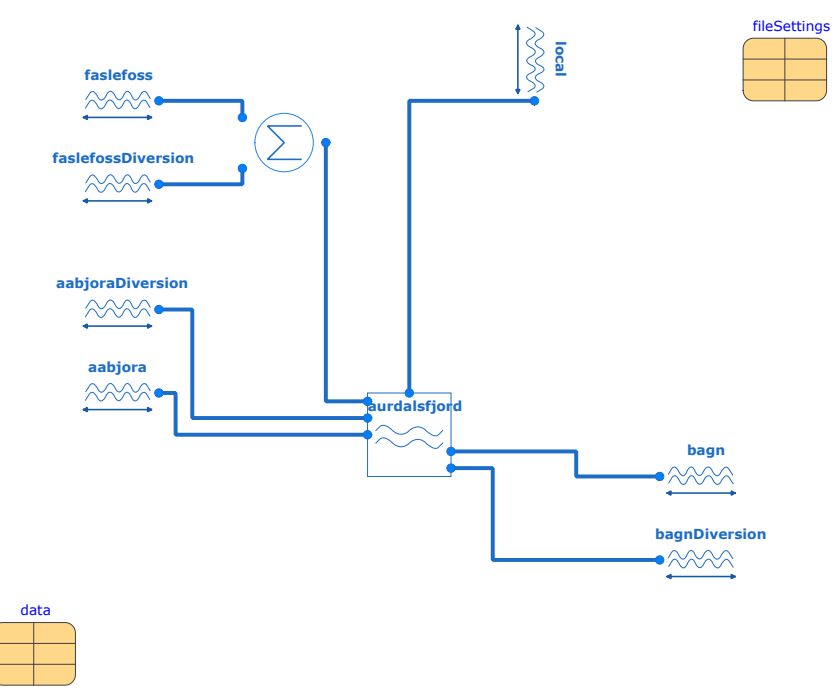

Figure 7. Aurdalsfjord implemented in Modelica.

defined by this parameter. Islands within the grid are possible and need to be given as zero-cells ( $0 \mathrm{~m}$ depth). Zerocells are also possible at the borders, however only if the border is not connected to an in- or outflow. Due to the local inflow being distributed over all borders, it is generally recommended to not have zero-cells at the borders.

The maximum water level is provided in $\mathrm{H} \_$max, which must not be misconceived as highest regulated water level (HRWL). The level is given as the maximum water level that can be simulated. It should be set higher than HRWL, so the simulation is able to cover situations with too high water levels.

The parameters $f \_p$ and $f \_M$ are factors that influence the weight of the pressure force and the incoming and outgoing momentum flows on the calculations. This is included in order to make the model work better for the Aurdalsfjord case, and is explained further in subsection 3.4. If there is no data to optimise against, the factors should be set to 1 .

$\mathrm{H}$-O defines the initial water level and applies for the entire reservoir. This was decided due to the model being limited to rectangular shapes. Hence, for more complex reservoirs, several Reservoir need to be connected and all of them can be contain $\mathrm{H} \_\mathrm{O}$ values.

\subsubsection{Basic Principles}

The cell structure within the Reservoir-model is based on the principles of the stacked grids. Models for the mass balance and for the momentum balance are developed. The models are based on the mathematical formulations in subsection 2.1 and subsection 2.2. In addition, a boundary-model is developed to control the in- and outflow of the reservoir.

A connector is developed to be able to send four values (water level, volume flow previous and next volume flows) from model to model. The mass balance model receives the volume flow and water levels across all four borders through the connector and calculates the wa- 

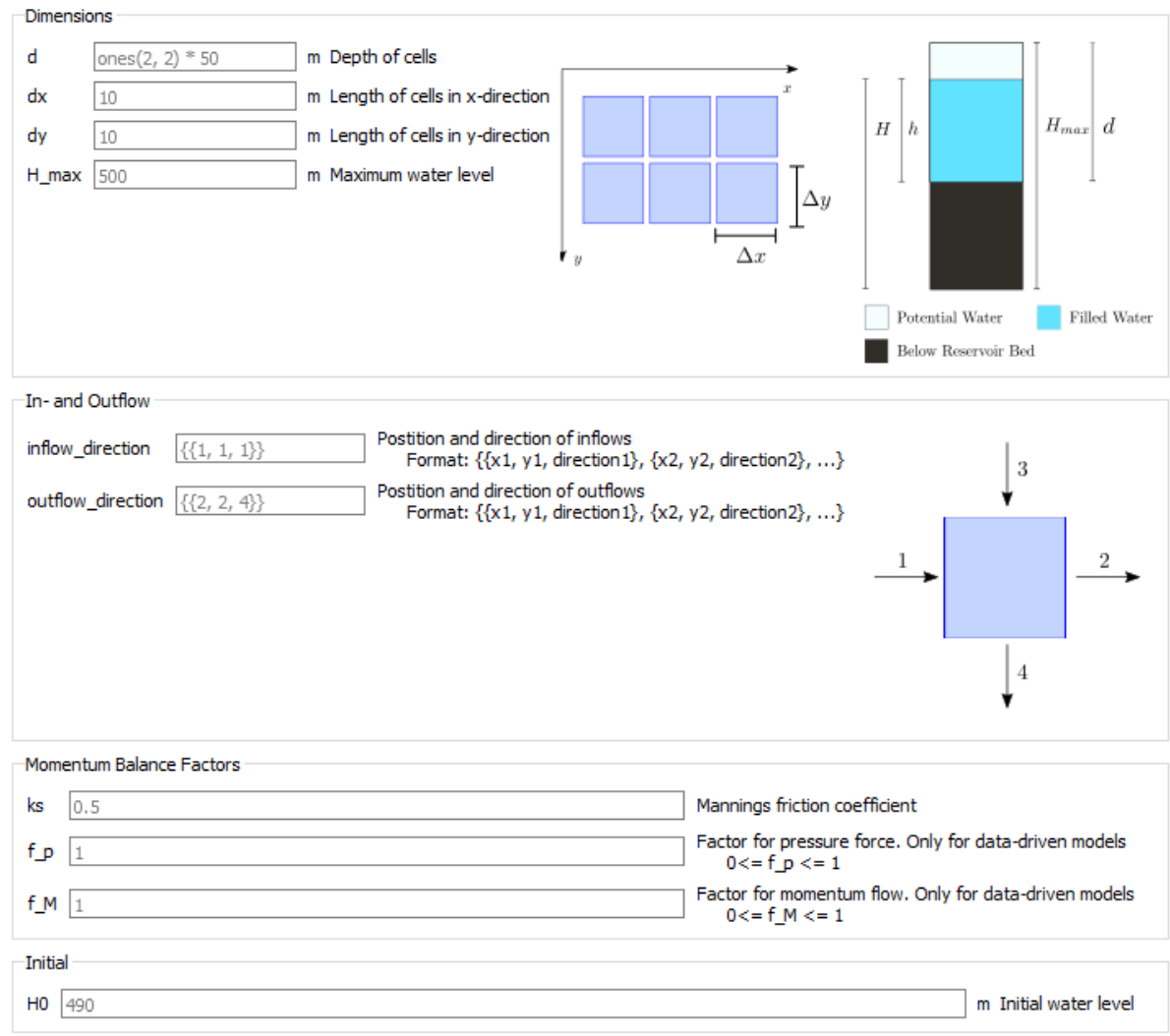

Figure 8. Reservoir-parameters.

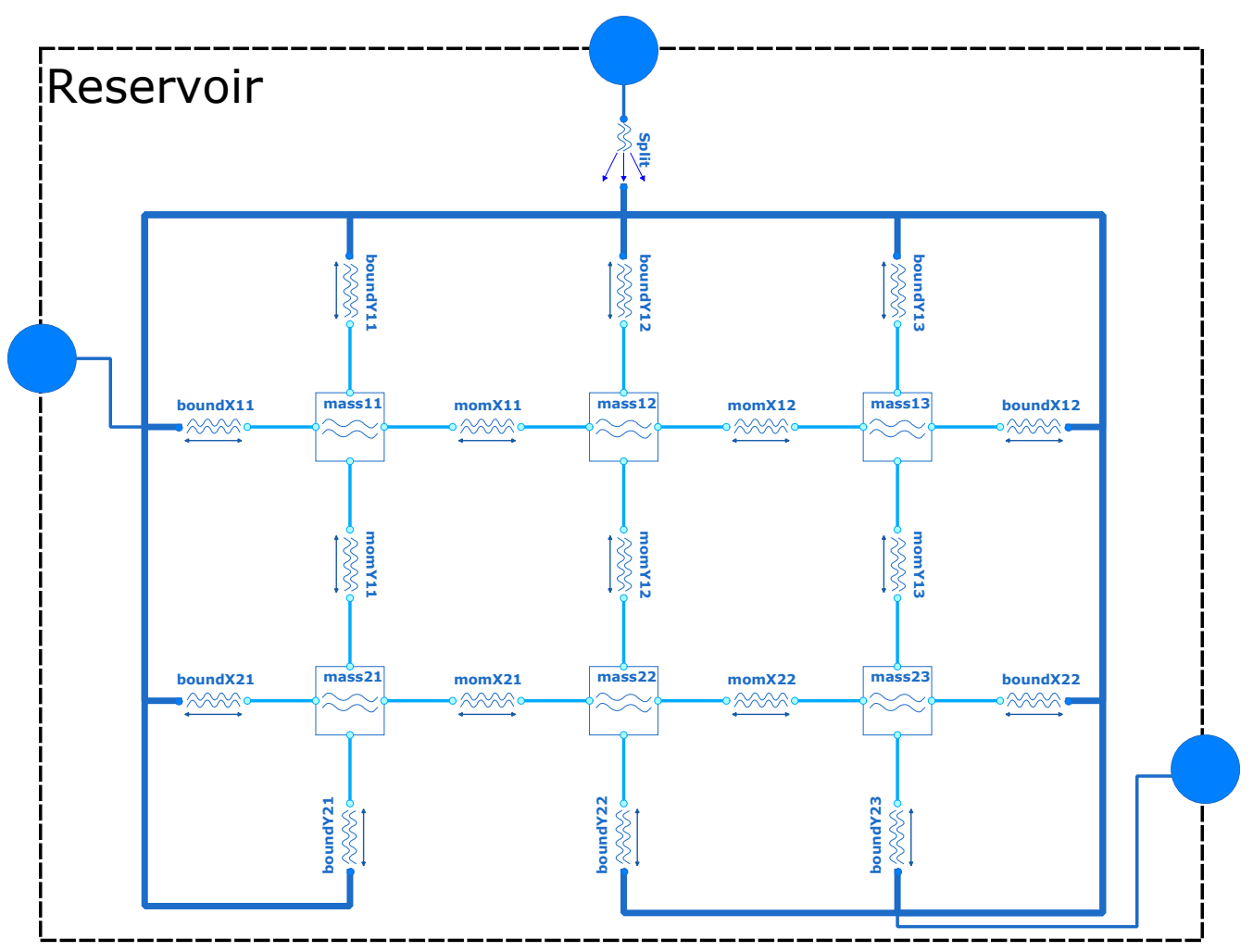

Figure 9. Grid structure in Reservoir. 
ter level. The momentum balance model needs the water levels of the connected mass balance models and the momentum flow of the adjacent momentum balance models. The boundary models decide the flow into the rest of the reservoir based on the connected in- and outflows.

The mass balance models are connected at all four borders to momentum balance models, except at the borders of the reservoir. Here, they are connected to boundarymodels. Each model in the grid structure calculates either a water level or a volume flow, and is always connected to a model that calculates the opposite. This principle was introduced in order for all models being independent on whether they are connected at a border or in the middle of the reservoir.

Figure 9 shows the inner workings of the Reservoir model. The blue circles at the edges are connectors which are connected to the inflow, outflow and local inflow (same as in Figure 7). They only send mass flows and pressures, which are translated in the boundary-models into water levels and volume flows.

The local inflow is split into equal parts and connected to all boundary-models (dark blue lines). If no in- or outflow is connected, Modelica views it as 0 flow. The inflow and outflow are connected to the parameter defined boundaries.

\subsection{Connect Multiple Reservoirs}

In cases where the reservoir has non-rectangular shapes, several Reservoir-models need to be connected. The models are connected at one of the in- or outflow connectors. Due to each Reservoir needing a flow value as the input, it is not possible to connect Reservoir-models directly. Another model that calculates the flow between them is necessary.

A ReservoirConnector model is developed. It is a simplified version of the momentum balance model. Due to the only values given through the connector being mass flow and pressure (can be transformed to volume flow and level), the incoming and outgoing momentum flows are unknown and set to zero. The rest of the calculations are identical to the momentum balance. In addition, the two connected cells (from each reservoir) need to have the same depth-value. It is possible to have several parallel connections between the same reservoirs. Otherwise each reservoir connection would introduce a bottleneck in he system. An example of two connected reservoirs is shown in Figure 10.

For Aurdalsfjord a total of 13 Reservoir-models are connected. They are shown in Figure 11a. The discretised grid is shown in Figure 11b.

\subsection{Hydro Power Plant Model}

The developed models can be included into full hydro power plant models together with models from OpenHPL. An example is shown in Figure 12.

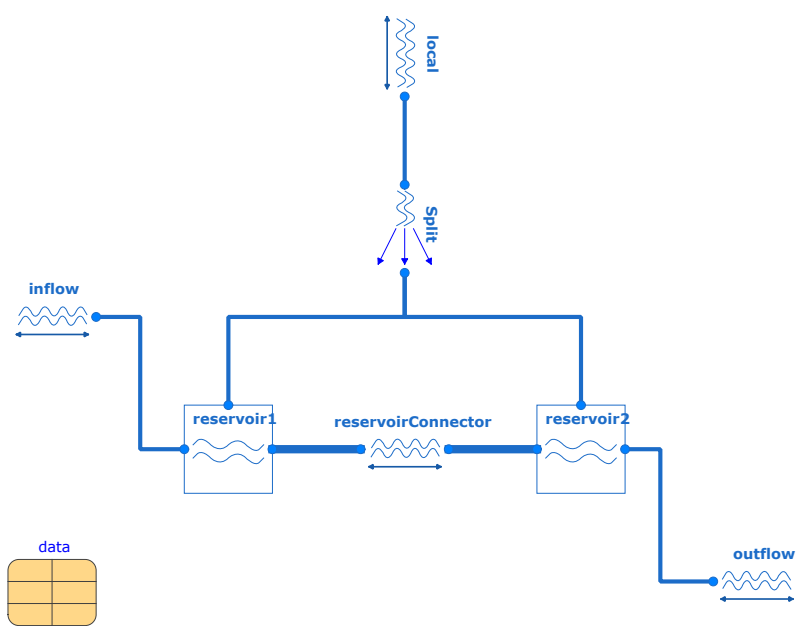

Figure 10. Two connected reservoirs in Modelica.

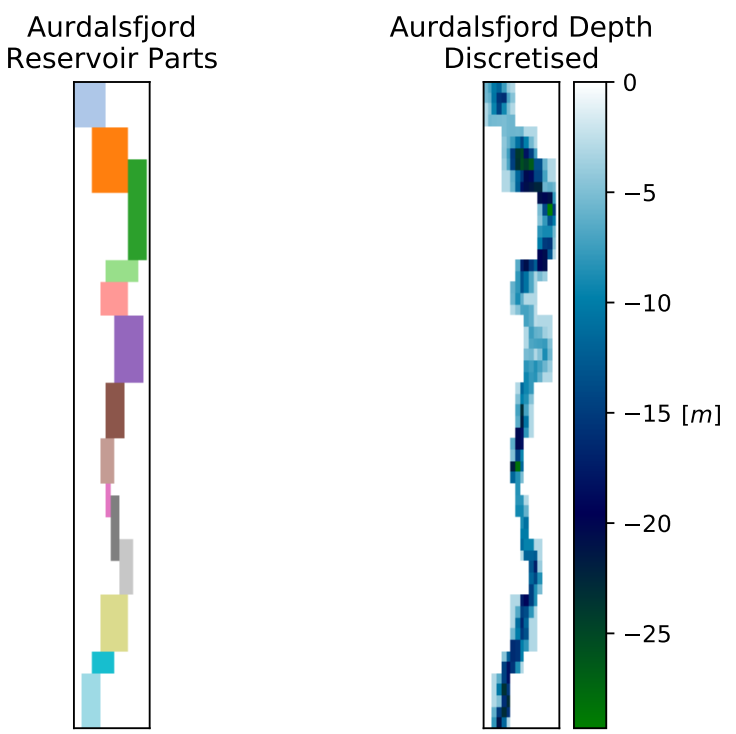

$\begin{array}{ll}\text { (a) Sub-reservoirs. } & \text { (b) Cells with depth values. }\end{array}$

Figure 11. Aurdalsfjord discretised into sub-reservoirs and cells.

\subsection{Simulations with Aurdalsfjord}

In the simulation results for Aurdalsfjord, the simulated and measured values for the two measurement stations are compared. One measurement station (Sundvoll) is located north of the narrowing, while the other is located at the dam south of the narrowing. All simulations are performed with actual in- and outflow data for winter periods in order to reduce the uncertainty caused by local inflow. The simulation step is set to $3600 \mathrm{~s}$ and the tolerance to 0.01 . The simulation time for 24 hours is approximately 1 hour.

The initial simulations for Aurdalsfjord proved unsuccessful, due to a spike in water level at the dam location 


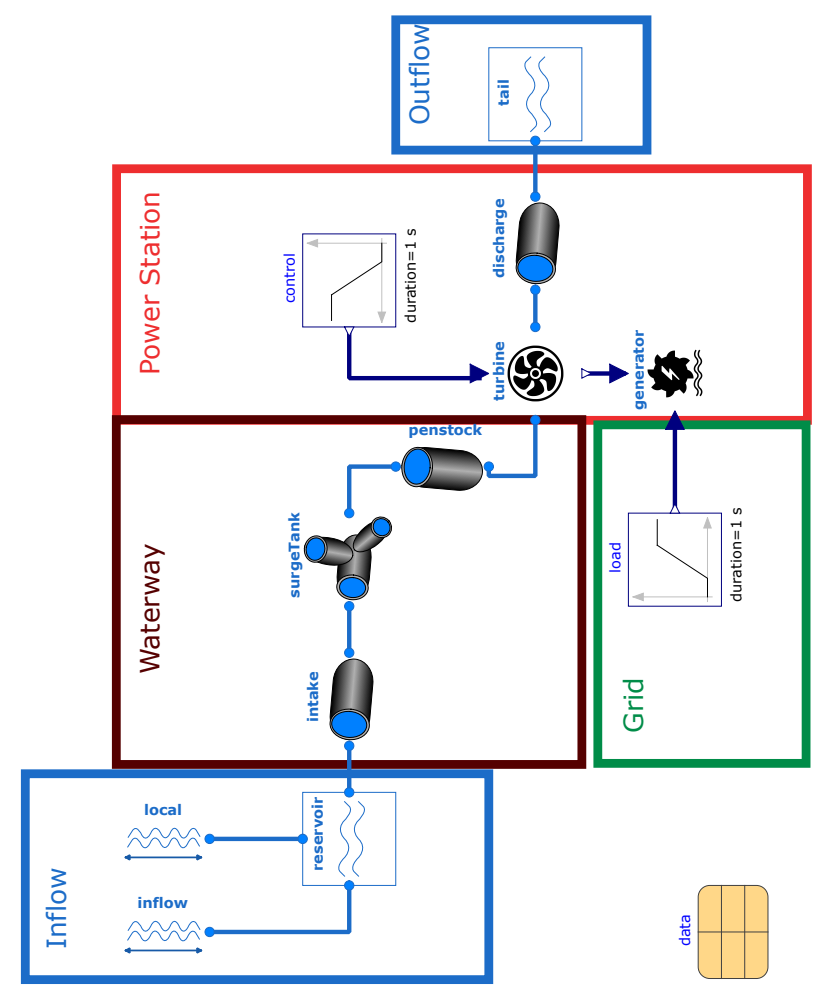

Figure 12. Reservoir-model integrated in hydro power plant model with OpenHPL-models.

leading to a simulation breakdown (shown in Figure 13a). This is solved by adding the additional parameters, $f_{\mathrm{M}}$ and $f_{\mathrm{p}}$ to the first equation in Equation 9, resulting in Equation 11 .

$$
\frac{\mathrm{d} M}{\mathrm{~d} t}=f_{\mathrm{M}}\left(\dot{M}_{1}-\dot{M}_{2}\right)+f_{\mathrm{M}} F_{\mathrm{p}}-F_{\mathrm{f}}
$$

With the additional factors, the parameters $k_{\mathrm{s}}, f_{\mathrm{p}}$ and $f_{\mathrm{M}}$ are estimated to be $0.136,1.0$ and 0.4 respectively. Figure $13 \mathrm{~b}$ shows the simulation result for the estimation period, while Figure 13c shows the simulation result for a different period using the same parameter values.

\section{Discussion}

\subsection{Implementation of Model}

Making a model as detailed and complex as possible is not necessarily better than keeping the model simple. More detailed models include more physical attributes of the actual system, however it will also increase the simulation time. An example is the choice to solely focus on horizontal flow. It would also be possible to include equations that calculate vertical flow in the reservoir, which would be more accurate to reality. The equation system becomes significantly more complex, as things like temperature need to be taken into account. However, water levels are not influenced by vertical flow, and the influence on the horizontal flow is minimal.

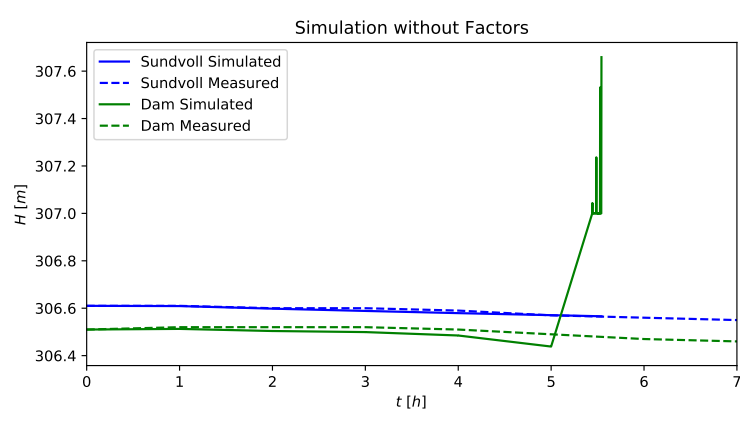

(a) Initial simulation.

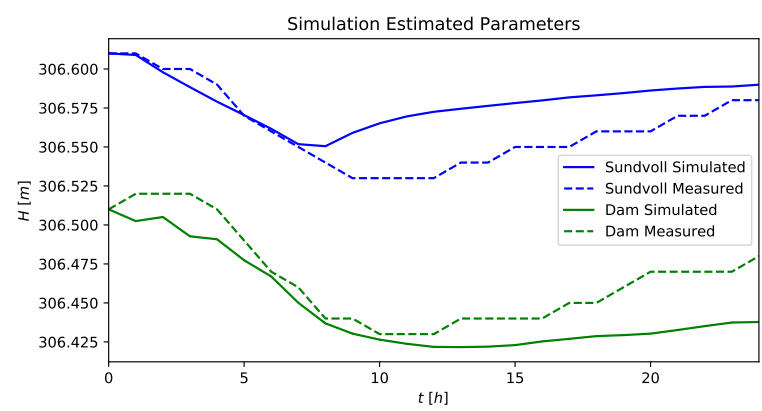

(b) Parameter estimation result.

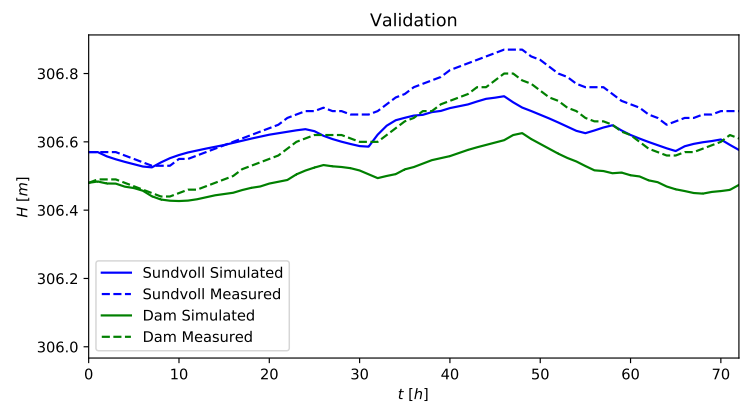

(c) Parameter validation.

Figure 13. Simulations showing simulated and measured values.

In the sum of forces in Equation 6, the pressure and friction forces are included. The gravity and Coriolis forces could also have been included. If the reservoir floor is described by a slope, the gravity that pushes the water down also pushes it partly forward (due to the slope). However, in the developed model, slopes are described as steps. In this case the gravity force only contains a vertical component. The momentum balance control volumes could also have been described with slopes, it was however decided not to include this, as most of the gravity force still only provides vertical flow. The gravity is however still included as part of the pressure force. The Coriolis force always points to the west due to the rotation of the earth. If included, the coordinate system in which the grid is given to the model, would need to be accurate according to earths coordinate system. The force would only be included in the flow in x-direction. The implementation of the force would not overly complicate the system, however due to Aurdalsfjord being narrow it was assumed 
that the effect on this exact case was minimal. It could be an option to include this in the future, especially if larger (wider) reservoirs are to be implemented.

Several simplifications are made with the implementation in Modelica, in order to keep the model simple. They are less significant if the chosen grid contains small cells. The two most significant are the connection of mass and momentum grids and the wetting perimeter in the friction force.

The momentum balance grid is on top of the mass balance grid, where each momentum control volume covers half of one mass control volume and half of the next mass control volume. This means that the mass flows in and out of the momentum control volume ( $\dot{m}_{\text {in }}$ and $\left.\dot{m}_{\text {out }}\right)$ should be the same as the mass flowing in the middle of the mass control volumes. In the model, the mass flows in and out of the momentum balance model are set equal to the mass flows in and out of the adjacent mass balance models. Hence, the mass flows are moved by half the length of one mass control volume.

For open channel flows (1-dimensional), the wetting perimeter $(\phi)$ in the friction force is normally set equal $2 h+\Delta w$. The $2 h$ part represents the friction against the side walls while the $\Delta w$ part represents the friction against the reservoir floor. In the model however, the $2 h$ part is dropped, due to it being assumed that most cells are in the middle of the reservoir without side walls. This neglects both the cells which are actually at the side of the reservoir and the fact that the reservoir floor is not at the same level through the reservoir. In some of the reservoir the water will flow parallel to a part with less depth and thereby have friction with a (low) side wall.

Instead of basing the model on the DAEs, the model could have been based on the Shallow Water Equations (SWE) (Plumb 2014). The equations are developed from the mass and momentum balance equations, where the cells are made infinitesimal small. Despite the SWE being used in many applications (Behrens 2017) it was chosen to use the DAE. DAEs are normally faster to solve in numeric calculations and Modelica is designed for implementation of DAEs. In addition, it is questionable if using PDEs are significantly more accurate due to larger reservoirs containing many uncertainties.

In OpenHPL there are models available for reservoir and open channel modelling (TMCC, USN 2020). The reservoir was not considered complex enough, due to it not modelling the dynamics within the reservoir (only the flow out). The open-channel models is implemented with the SWE as PDEs for one directional flow. This could have been extended to two directional flow and used as an initial building block, instead of the mass and momentum balances.

\subsection{Simulation Results}

Figure 13a shows that with both new factors set to 1 , the water level at the dam starts spiking the momentum the level increases fast and leads to a breakdown of the simu- lation. The estimated parameters show that the influence of momentum flow on the momentum balance should be reduced significantly. Both of these statements indicate that the reservoir is responsive to large flows. The fact that only the dam level starts behaving unrealistic also indicates that the narrowing might be part of the problem. A reason might be that the generic model is designed for a reservoir where most cells are in the middle of the reservoir (without borders), while in the narrowing all cells are at the borders.

The estimated parameters show that the pressure factor should stay around 1, while the momentum factor influences the system more significantly. It could be considered to remove the pressure factor from the model, which would force it to 1 .

The results in Figure 13b and Figure 13c show that the model is able to simulate realistic results when the parameters are estimated for the same period. However, when the same parameters are used for a different simulation period, the results are less accurate. Due to the time spent on the parameter estimation is limited, it could be possible to improve them and thereby possibly improve the simulation results.

\section{Conclusion}

The Modelica model shows promising results. For Aurdalsfjord, a reservoir with a narrowing between in- and outflow, it needed to be adjusted with non-physical parameters. They reduce the weight of the pressure force and the momentum flows in the momentum balance. The reservoir model should be implemented for a less complex shaped reservoir in order to see if the challenges are related to the Modelica implementation or Aurdalsfjords shape.

The new reservoir model presented in this paper will be part of a future version of OpenHPL.

\section{References}

Jörn Behrens. 2017. Surprisingly Rich in Applications: The Shallow Water Equations and Numerical Solution Methods. URL: https://sinews.siam. org / Details-Page / surprisingly-rich-inapplications - the - shallow - waterequations - and - numerical - solution methods [Accessed 16 January 2020].

Dassault Systèmes. Dymola. 2020. URL: https : / / www. 3ds.com/products-services/catia/ products/dymola/ [Accessed 9 June 2020].

Bernt Lie. Modelling of Dynamic Systems, 247 pages, 2017.

Willem Meijer. Dynamic Reservoir Model Using Modelica: Modelling of Water Levels in Aurdalsfjord. M.Sc.Thesis, University of SouthEastern Norway, Porsgrunn. 121 pages, 2020.

Open-Source Modelica Consortium. OMPython - A Python Interface to OpenModelica Communicating via CORBA or ZeroMQ. 2020a. OpenModelica. 
URL: https: / / github. com/OpenModelica/

OMPython [Accessed 24 February 2020].

Open-Source Modelica Consortium. OpenModelica.

(2020b) URL: https : / / openmodelica . org

[Accessed 24 February 2020].

Alan Plumb. (2014). Dynamics of the Atmosphere -

Lecture Notes. Chapter 2: The Shallow Water

Equations. Massachusetts Institute of Technology.

URL: http : / / www-aps . mit. edu / rap /

courses / 12333_notes / A2 \% 20SWeqs . pdf

[Accessed 15 February 2020].

TMCC, USN. OpenHPL - an Open-Source Hydropower Library That Consists of Hydropower Unit Models.

2020 URL: https : openhpl . simulati.no

[Accessed 24 February 2020]. 